\title{
The Korteweg-de Vries equation and a Diophantine problem related to Bernoulli polynomials
}

\author{
Ákos Pintér ${ }^{1 *}$ and Szabolcs Tengely ${ }^{2}$ \\ Dedicated to Professor Hari M Srivastava
}

${ }^{*}$ Correspondence:

apinter@science.unideb.hu

${ }^{1}$ Institute of Mathematics, MTA-DE

Research Group 'Equations,

Functions and Curves', Hungarian

Academy of Sciences, University of

Debrecen, P.O. Box 12, Debrecen,

4010, Hungary

Full list of author information is

available at the end of the article

\begin{abstract}
Some Diophantine equations related to the soliton solutions of the Korteweg-de Vries equation are resolved. The main tools are the connection with Bernoulli polynomials and the application of certain computational number-theoretical results. MSC: Primary 11D41; 14H45; secondary 11Y50
\end{abstract}

Keywords: Diophantine equations; curves of genus 2; Korteweg-de Vries equation

\section{Introduction}

In the paper [1], Fairlie and Veselov obtained a relation of the Bernoulli polynomials with the theory of the Korteweg-de Vries (KdV) equation

$$
u_{t}-6 u u_{x}+u_{x x x}=0 .
$$

This equation has infinitely many conservation laws (that is, certain laws, which show that a particular measurable property of an isolated physical system, like mass, energy, momentum, etc., does not change as the system evolves) of the form

$$
I_{m}[u]=\int_{-\infty}^{\infty} P_{m}\left(u, u_{x}, u_{x x}, \ldots, u_{m}\right) d x,
$$

where $P_{m}$ are some polynomials of the function $u$ and its $x$-derivatives up to order $m$, see [2]. For example,

$$
I_{-1}[u]=\int_{-\infty}^{\infty} u d x, \quad I_{0}[u]=\int_{-\infty}^{\infty} u^{2} d x, \quad I_{1}[u]=\int_{-\infty}^{\infty}\left(u_{x}^{2}+2 u^{3}\right) d x
$$

and

$$
I_{2}[u]=\int_{-\infty}^{\infty}\left(u_{x x}^{2}+10 u u_{x}^{2}+5 u^{4}\right) d x
$$

The $\mathrm{KdV}$ equation possesses a remarkable family of so-called $n$-soliton solutions corresponding to the initial profile $u_{n}(x, 0)=-2 n(n+1) \operatorname{sech}^{2} x$. For some recent generalizations

C) 2013 Pintér and Tengely; licensee Springer. This is an Open Access article distributed under the terms of the Creative Commons Attribution License (http://creativecommons.org/licenses/by/2.0), which permits unrestricted use, distribution, and reproduction in any medium, provided the original work is properly cited. 
and applications of the Korteweg-de Vries equation, we refer to [3, 4] and [5] and the references given therein.

Using the spectral theory of Schrödinger operators, see [6], Fairlie and Veselov [1] proved that there is a strong connection between the improver integrals related to the functions $u_{n}(x, 0)$ above and the well-known discrete sums of power values, namely,

$$
I_{k}\left[u_{n}\right]=\frac{(-1)^{k} 4^{k+2}}{2 k+3} \sum_{i=1}^{n} i^{2 k+3}
$$

for $k=-1,0,1, \ldots$

Now, let $k \neq l$ be fixed integers with $k, l \in\{-1,0,1,2, \ldots\}$, and suppose that

$$
\left|I_{k}\left[u_{n}\right]\right|=\left|I_{l}\left[u_{m}\right]\right|
$$

One can ask how often can these integrals be equal for given $k$ and $l$ ? In other words, what is the cardinality of the set of solutions $m, n$ to the equation

$$
\frac{4^{k}}{2 k+3} \sum_{i=1}^{n} i^{2 k+3}=\frac{4^{l}}{2 l+3} \sum_{i=1}^{m} i^{2 l+3}
$$

where $k$ and $l$ are fixed distinct integers? Of course, one can consider the much more general problem, when $k$ and $l$ are also unknown integers; however, in this case, the solution of the corresponding equation seems beyond the reach of current techniques.

Applying some recent results by Rakaczki, see [7] and [8], it is not too hard to give some ineffective and effective finiteness statements for the solutions $m$ and $n$ to equation (1). However, the purpose of this note is to resolve (1) for certain values of $m$ and $n$, including an infinite family of the parameters.

Theorem 1 For $k=-1$ and $l \in\{0,1,2,3\}$, equation (1) has only one solution, namely, $(l, m, n)=(0,24,5)$.

Theorem 2 Assume that $k=0$ and $l$ is a positive integer such that $2 l+3$ is prime. Then (1) has no solution in positive integers $m$ and $n$.

\section{Auxiliary results}

In our first lemma, we summarize some classical properties of Bernoulli polynomials. For the proofs of these results, we refer to [9].

Lemma 1 Let $B_{j}(X)$ denote the $j$ th Bernoulli polynomial and $B_{j}=B_{j}(0), j=1,2, \ldots$. Further, let $D_{j}$ be the denominator of $B_{j}$. Then we have

(A) $B_{j}(X)=X^{n}+\sum_{i=1}^{j}\left(\begin{array}{l}j \\ i\end{array}\right) B_{i} X^{j-i}$,

(B) $S_{j}(x)=1^{j}+2^{j}+\cdots+(x-1)^{j}=\frac{1}{j+1}\left(B_{j+1}(x)-B_{j+1}\right)$,

(C) $B_{1}=-\frac{1}{2}, B_{2 j+1}=0, j=1,2, \ldots$,

(D) (von Staudt-Clausen) $D_{2 j}=\prod_{p-1 \mid 2 j, p \text { prime }} p$,

(E) $X^{2}(X-1)^{2} \mid B_{2 j}(X)-B_{2 j}($ in $\mathbb{Q}[X])$,

(F) $B_{j}(X)=(-1)^{j} B_{j}(1-X)$. 
Consider the hyperelliptic curve

$$
\mathcal{C}: y^{2}=F(x):=x^{5}+b_{4} x^{4}+b_{3} x^{3}+b_{2} x^{2}+b_{1} x+b_{0}
$$

where $b_{i} \in \mathbb{Z}$. Let $\alpha$ be a root of $F$, and let $J(\mathbb{Q})$ be the Jacobian of the curve $\mathcal{C}$. We have that

$$
x-\alpha=\kappa \xi^{2},
$$

where $\kappa, \xi \in K=\mathbb{Q}(\alpha)$ and $\kappa$ comes from a finite set. By knowing the Mordell-Weil group of the curve $\mathcal{C}$, it is possible to provide a method to compute such a finite set. To each coset representative $\sum_{i=1}^{m}\left(P_{i}-\infty\right)$ of $J(\mathbb{Q}) / 2 J(\mathbb{Q})$, we associate

$$
\kappa=\prod_{i=1}^{m}\left(\gamma_{i}-\alpha d_{i}^{2}\right)
$$

where the set $\left\{P_{1}, \ldots, P_{m}\right\}$ is stable under the action of Galois, all $y\left(P_{i}\right)$ are non-zero and $x\left(P_{i}\right)=\gamma_{i} / d_{i}^{2}$, where $\gamma_{i}$ is an algebraic integer and $d_{i} \in \mathbb{Z}_{\geq 1}$. If $P_{i}, P_{j}$ are conjugate, then we may suppose that $d_{i}=d_{j}$, and so, $\gamma_{i}, \gamma_{j}$ are conjugate. We have the following lemma (Lemma 3.1 in [10]).

Lemma 2 Let $\mathcal{K}$ be a set of $\kappa$ values, associated as above to a complete set of coset representatives of $J(\mathbb{Q}) / 2 J(\mathbb{Q})$. Then $\mathcal{K}$ is a finite subset of $\mathcal{O}_{K}$, and if $(x, y)$ is an integral point on the curve (2), then $x-\alpha=\kappa \xi^{2}$ for some $\kappa \in \mathcal{K}$ and $\xi \in K$.

As an application of his theory of lower bounds for linear forms in logarithms, Baker [11] gave an explicit upper bound for the size of integral solutions of hyperelliptic curves. This result has been improved by many authors (see, e.g., [12-18] and [19]).

In [10], an improved completely explicit upper bound were proved combining ideas from $[15,19-25]$. Now we will state the theorem, which gives the improved bound. We introduce some notation. Let $K$ be a number field of degree $d$, and let $r$ be its unit rank, and let $R$ be its regulator. For $\alpha \in K$, we denote by $\mathrm{h}(\alpha)$ the logarithmic height of the element $\alpha$. Let

$$
\partial_{K}= \begin{cases}\frac{\log 2}{d} & \text { if } d=1,2, \\ \frac{1}{4}\left(\frac{\log \log d}{\log d}\right)^{3} & \text { if } d \geq 3,\end{cases}
$$

and let

$$
\partial_{K}^{\prime}=\left(1+\frac{\pi^{2}}{\partial_{K}^{2}}\right)^{1 / 2} .
$$

Define the constants

$$
\begin{array}{ll}
c_{1}(K)=\frac{(r !)^{2}}{2^{r-1} d^{r}}, \quad c_{2}(K)=c_{1}(K)\left(\frac{d}{\partial_{K}}\right)^{r-1}, \\
c_{3}(K)=c_{1}(K) \frac{d^{r}}{\partial_{K}}, & c_{4}(K)=r d c_{3}(K), \quad c_{5}(K)=\frac{r^{r+1}}{2 \partial_{K}^{r-1}} .
\end{array}
$$


Let

$$
\partial_{L / K}=\max \left\{[L: \mathbb{Q}],[K: \mathbb{Q}] \partial_{K}^{\prime}, \frac{0.16[K: \mathbb{Q}]}{\partial_{K}}\right\},
$$

where $K \subseteq L$ are number fields. Define

$$
C(K, n):=3 \cdot 30^{n+4} \cdot(n+1)^{5.5} d^{2}(1+\log d) .
$$

The following result will be used to get an upper bound for the size of the integral solutions of our equations. It is Theorem 3 in [10].

Lemma 3 Let $\alpha$ be an algebraic integer of degree at least 3 , and let $\kappa$ be an integer belonging to $K$. Denote by $\alpha_{1}, \alpha_{2}, \alpha_{3}$ distinct conjugates of $\alpha$ and by $\kappa_{1}, \kappa_{2}, \kappa_{3}$ the corresponding conjugates of $\kappa$. Let

$$
K_{1}=\mathbb{Q}\left(\alpha_{1}, \alpha_{2}, \sqrt{\kappa_{1} \kappa_{2}}\right), \quad K_{2}=\mathbb{Q}\left(\alpha_{1}, \alpha_{3}, \sqrt{\kappa_{1} \kappa_{3}}\right), \quad K_{3}=\mathbb{Q}\left(\alpha_{2}, \alpha_{3}, \sqrt{\kappa_{2} \kappa_{3}}\right)
$$

and

$$
L=\mathbb{Q}\left(\alpha_{1}, \alpha_{2}, \alpha_{3}, \sqrt{\kappa_{1} \kappa_{2}}, \sqrt{\kappa_{1} \kappa_{3}}\right) .
$$

In what follows $R$ stands for an upper bound for the regulators of $K_{1}, K_{2}$ and $K_{3}$, and $r$ denotes the maximum of the unit ranks of $K_{1}, K_{2}, K_{3}$. Let

$$
c_{j}^{*}=\max _{1 \leq i \leq 3} c_{j}\left(K_{i}\right),
$$

and let

$$
N=\max _{1 \leq i, j \leq 3}\left|\operatorname{Norm}_{\mathbb{Q}\left(\alpha_{i}, \alpha_{j}\right) / \mathbb{Q}}\left(\kappa_{i}\left(\alpha_{i}-\alpha_{j}\right)\right)\right|^{2},
$$

and let

$$
H^{*}=c_{5}^{*} R+\frac{\log N}{\min _{1 \leq i \leq 3}\left[K_{i}: \mathbb{Q}\right]}+\mathrm{h}(\kappa) .
$$

Define

$$
A_{1}^{*}=2 H^{*} \cdot C(L, 2 r+1) \cdot\left(c_{1}^{*}\right)^{2} \partial_{L / L} \cdot\left(\max _{1 \leq i \leq 3} \partial_{L / K_{i}}\right)^{2 r} \cdot R^{2}
$$

and

$$
A_{2}^{*}=2 H^{*}+A_{1}^{*}+A_{1}^{*} \log \left\{(2 r+1) \cdot \max \left\{c_{4}^{*}, 1\right\}\right\} .
$$

If $x \in \mathbb{Z} \backslash\{0\}$ satisfies $x-\alpha=\kappa \xi^{2}$ for some $\xi \in K$ then

$$
\log |x| \leq 8 A_{1}^{*} \log \left(4 A_{1}^{*}\right)+8 A_{2}^{*}+H^{*}+20 \log 2+13 \mathrm{~h}(\kappa)+19 \mathrm{~h}(\alpha) .
$$


To obtain a lower bound for the possible unknown integer solutions, we are going to use the so-called Mordell-Weil sieve. The Mordell-Weil sieve has been successfully applied to prove the non-existence of rational points on curves (see, e.g., [26-28] and [29]).

Let $C / \mathbb{Q}$ be a smooth projective curve (in our case a hyperelliptic curve) of genus $g \geq 2$. Let $J$ be its Jacobian. We assume the knowledge of some rational point on $C$, so let $D$ be a fixed rational point on $C$, and let $J$ be the corresponding Abel-Jacobi map

$$
J: C \rightarrow J, \quad P \mapsto[P-D] .
$$

Let $W$ be the image in $J$ of the known rational points on $C$ and $D_{1}, \ldots, D_{r}$ generators for the free part of $J(\mathbb{Q})$. By using the Mordell-Weil sieve, we are going to obtain a very large and smooth integer $B$ such that

$$
J(C(\mathbb{Q})) \subseteq W+B J(\mathbb{Q}) .
$$

Let

$$
\phi: \mathbb{Z}^{r} \rightarrow J(\mathbb{Q}), \quad \phi\left(a_{1}, \ldots, a_{r}\right)=\sum a_{i} D_{i}
$$

so that the image of $\phi$ is the free part of $J(\mathbb{Q})$. The variant of the Mordell-Weil sieve explained in [10] provides a method to obtain a very long decreasing sequence of lattices in $\mathbb{Z}^{r}$

$$
B \mathbb{Z}^{r}=L_{0} \supsetneq L_{1} \supsetneq L_{2} \supsetneq \cdots \supsetneq L_{k}
$$

such that

$$
J(C(\mathbb{Q})) \subset W+\phi\left(L_{j}\right)
$$

for $j=1, \ldots, k$.

The next lemma [10, Lemma 12.1] gives a lower bound for the size of rational points, whose images are not in the set $W$.

Lemma 4 Let $W$ be a finite subset of $J(\mathbb{Q})$, and let $L$ be a sublattice of $\mathbb{Z}^{r}$. Suppose that $J(C(\mathbb{Q})) \subset W+\phi(L)$. Let $\mu_{1}$ be a lower bound for $h-\hat{h}$ and

$$
\mu_{2}=\max \{\sqrt{\hat{h}(w)}: w \in W\} .
$$

Denote by $M$ the height-pairing matrix for the Mordell-Weil basis $D_{1}, \ldots, D_{r}$, and let $\lambda_{1}, \ldots, \lambda_{r}$ be its eigenvalues. Let

$$
\mu_{3}=\min \left\{\sqrt{\lambda_{j}}: j=1, \ldots, r\right\},
$$

and let $m(L)$ be the Euclidean norm of the shortest non-zero vector of $L$. Then, for any $P \in$ $C(\mathbb{Q})$, either $J(P) \in W$ or

$$
h(J(P)) \geq\left(\mu_{3} m(L)-\mu_{2}\right)^{2}+\mu_{1} .
$$


The following lemma plays a crucial role in the proof of Theorem 1.

Lemma 5 The integral solutions of the equation

$$
\mathcal{C}: Y^{2}=X(X+20)^{2}\left(X^{2}+10 X+400\right)+140,625
$$

are

$$
(X, Y) \in\{(0, \pm 375),(-20, \pm 375)\} .
$$

Proof of Lemma 5 Let $J(\mathbb{Q})$ be the Jacobian of the genus two curve (3). Using MAGMA, we determine a Mordell-Weil basis, which is given by

$$
\begin{aligned}
& D_{1}=(0,375)-\infty, \\
& D_{2}=(-20,375)-\infty .
\end{aligned}
$$

Let $f=x(x+20)^{2}\left(x^{2}+10 x+400\right)+140,625$, and let $\alpha$ be a root of $f$. We will choose for coset representatives of $J(\mathbb{Q}) / 2 J(\mathbb{Q})$ the linear combinations $\sum_{i=1}^{2} n_{i} D_{i}$, where $n_{i} \in\{0,1\}$. Then

$$
x-\alpha=\kappa \xi^{2},
$$

where $\kappa \in \mathcal{K}$, and $\mathcal{K}$ is constructed as described in Lemma 2. We have that $\mathcal{K}=$ $\{1,-\alpha,-20-\alpha, \alpha(\alpha+20)\}$. By local arguments, it is possible to restrict the set $\mathcal{K}$ further (see, e.g., $[26,30])$. In our case, one can eliminate

$$
\alpha(\alpha+20)
$$

by local computations in $\mathbb{Q}_{3}$. We apply Lemma 3 to get a large upper bound for $\log |x|$ in the remaining cases. A MAGMA code was written to obtain the bounds that appeared in [10]; they can be found at http://www.warwick.ac.uk/ maseap/progs/intpoint/bounds.m. We obtain that these bounds are as in Table 1.

The set of known rational points on the curve (3) is $\{\infty,(0, \pm 375),(-20, \pm 375)\}$. Let $W$ be the image of this set in $J(\mathbb{Q})$. Applying the Mordell-Weil sieve, implemented by Bruin and Stoll and explained in $[10]$, we obtain that $J(C(\mathbb{Q})) \subseteq W+B J(\mathbb{Q})$, where

$$
B=2^{8} \cdot 5^{3} \cdot 7^{2} \cdot 11^{2} \cdot 13^{2} \cdot 17^{2} \cdot 19 \cdot 31 \cdot 37 \cdot 41 \cdot 43 \cdot 53 \cdot 59 \cdot 71 \cdot 79 \cdot 83 \cdot 89
$$

that is

$$
B=46,128,223,306,000,188,203,435,897,312,000 .
$$

\section{Table 1 Bounds}

\begin{tabular}{ll}
\hline $\boldsymbol{\kappa}$ & Bound for $\log |\boldsymbol{x}|$ \\
\hline 1 & $6.27 \cdot 10^{307}$ \\
$-\alpha$ & $4.48 \cdot 10^{668}$ \\
$-20-\alpha$ & $1.89 \cdot 10^{612}$ \\
\hline
\end{tabular}


Now, we use an extension of the Mordell-Weil sieve due to Samir Siksek to obtain a very long decreasing sequence of lattices in $\mathbb{Z}^{2}$. After that, we apply Lemma 4 to obtain a lower bound for possible unknown rational points. We get that if $(x, y)$ is an unknown integral point, then

$$
\log |x| \geq 2.216448 \times 10^{782}
$$

This contradicts the bound for $\log |x|$ that we obtained by Baker's method.

\section{Proofs of the theorems}

Proof of Theorem 1 For $k=-1$ and $l \in\{0,1,2,3\}$, we have the Diophantine equations

$$
\begin{aligned}
& \frac{n(n+1)}{2}=\frac{m^{2}(m+1)^{2}}{3}, \\
& \frac{n(n+1)}{8}=\frac{1}{15} z^{2}(2 z-1) \quad \text { with } z=m(m+1), \\
& \frac{n(n+1)}{8}=\frac{2}{21} z^{2}\left(3 z^{2}-4 z+2\right) \quad \text { with } z=m(m+1)
\end{aligned}
$$

and

$$
\frac{1}{4} \sum_{i=1}^{n} i=\frac{64}{9} \sum_{i=1}^{m} i^{9},
$$

respectively. One can see that the first three equations are elliptic Diophantine equations, thus using the program package MAGMA, subroutines IntegralPoints or IntegralQuarticPoints are just a straightforward calculation to solve them. In these cases, the unique solution is $(l, m, n)=(0,24,5)$. The forth equation can be written as follows

$$
(2 n+1)^{2}=\frac{128}{45}\left(m^{2}+m-1\right)\left(m^{2}+m\right)^{2}\left(2 m^{4}+4 m^{3}-m^{2}-3 m+3\right)+1 .
$$

So, we easily obtain a hyperelliptic curve

$$
Y^{2}=X(X+20)^{2}\left(X^{2}+10 X+400\right)+140,625,
$$

where $Y=375(2 n+1)$ and $X=20 m^{2}+20 m-20$. By Lemma 5, we have that $X=0$ or -20 . Therefore, we have that $m \in\{-1,0\}$, a contradiction and there is no solution in positive integers of (7).

Proof of Theorem 2 Now $k=0$ and $p=2 l+3 \geq 3$ is a prime. From (1), we get

$$
p \cdot n^{2}(n+1)^{2}=3 \cdot 4^{l+1}\left(1^{p}+2^{p}+\cdots+m^{p}\right) .
$$

Let $m$ and $n$ be an arbitrary but fixed solution. An elementary number theoretical argument and Lemma 1 yield that $p \mid m(m+1)$ and

$$
\operatorname{ord}_{p}\left(\frac{1^{p}+2^{p}+\cdots+m^{p}}{m^{2}(m+1)^{2}}\right)=\operatorname{ord}_{p} \frac{B_{p+1}(m+1)-B_{p+1}}{m^{2}(m+1)^{2}} \neq 0 .
$$


Suppose that $p \mid m$, and let $d$ be the smallest positive integer such that $B_{p+1}(m+1)-B_{p+1}=$ $\frac{1}{d} f(m) m^{2}(m+1)^{2}$, and let $f(X) \in \mathbb{Z}[X]$. Since $\left(\begin{array}{c}p+1 \\ k\end{array}\right)$ is divisible by $p$ for $k=2, \ldots, p-1$ and $B_{1}=-1 / 2$, we have that $p$ is not a divisor of $d$. The constant term of the polynomial $f(X)$ is $d\left(\begin{array}{c}p+1 \\ p-1\end{array}\right) B_{p-1}$, and, by von Staudt-Clausen theorem, it is not divisible by $p$. On the other hand, $p$ is a divisor of $m$ and $f(m)$, we have a contradiction. If $p \mid m+1$, then we can repeat the previous argument using the fact $f(X)=f(-X-1), c f$. Lemma 1 .

Competing interests

The authors declare that they have no competing interests.

\section{Authors' contributions}

The authors have made equal contributions to this manuscript. All authors read and approved the final version.

\section{Author details}

'Institute of Mathematics, MTA-DE Research Group 'Equations, Functions and Curves', Hungarian Academy of Sciences, University of Debrecen, P.O. Box 12, Debrecen, 4010, Hungary. ${ }^{2}$ Institute of Mathematics, University of Debrecen, P.O. Box 12, Debrecen, 4010, Hungary.

\section{Acknowledgements}

The work is supported by the TÁMOP-4.2.2.C-11/1/KONV-2012-0010 project. The project is implemented through the New Hungary Development Plan, co-financed by the European Social Fund and the European Regional Development Fund. Research was supported in part by the Hungarian Academy of Sciences, OTKA grants K75566, K100339, NK101680, NK104208 (ÁP) and OTKA grants PD75264, NK104208, K100339 and János Bolyai Research Scholarship of the Hungarian Academy of Sciences (ST). The authors are grateful to the reviewers for their helpful remarks.

Received: 19 April 2013 Accepted: 29 July 2013 Published: 14 August 2013

\section{References}

1. Fairlie, DB, Veselov, AP: Faulhaber and Bernoulli polynomials and solitons. Physica D 152-153, 47-50 (2001)

2. Miura, RM, Gardner, CS, Kruskal, WD: Korteweg-de Vries equation and generalizations. II. J. Math. Phys. 9, 1204-1209 (1968)

3. Ismail, MS: Numerical solution of complex modified Korteweg-de Vries equation by Petrov-Galerkin method. Appl. Math. Comput. 202, 520-531 (2008)

4. Gai, X-L, Gao, Y-T, Yu, X, Wang, L: Painlevé property, auto-Bäcklund transformation and analytic solutions of a variable-coefficient modified Korteweg-de Vries model in a hot magnetized dusty plasma with charge fluctuations. Appl. Math. Comput. 216, 271-279 (2011)

5. Rady, ASA, Khater, AH, Osman, ES, Khafallah, M: New periodic wave and soliton solutions for system of coupled Korteweg-de Vries equations. Appl. Math. Comput. 207, 406-414 (2009)

6. Zakharov, VE, Faddaev, LD: KdV equation is completely integrable Hamiltonian system. Funct. Anal. Appl. 5, 18-27 (1971)

7. Rakaczki, C: On the Diophantine equation $S_{m}(x)=g(y)$. Publ. Math. (Debr.) $65,439-460$ (2004)

8. Rakaczki, C: On some generalization of the Diophantine equation $s\left(1^{k}+2^{k}+\cdots+x^{k}\right)+r=d y^{n}$. Acta Arith. 151, 201-216 (2012)

9. Rademacher, H: Topics in Analytic Number Theory. Springer, Berlin (1973)

10. Bugeaud, Y, Mignotte, M, Siksek, S, Stoll, M, Tengely, S: Integral points on hyperelliptic curves. Algebra Number Theory 2, 859-885 (2008)

11. Baker, A: Bounds for the solutions of the hyperelliptic equation. Proc. Camb. Philos. Soc. 65, 439-444 (1969)

12. Bilu, YF: Effective analysis of integral points on algebraic curves. Isr. J. Math. 90, 235-252 (1995)

13. Bilu, YF, Hanrot, G: Solving superelliptic Diophantine equations by Baker's method. Compos. Math. 112, 273-312 (1998)

14. Brindza, B: On S-integral solutions of the equation $y^{m}=f(x)$. Acta Math. Hung. 44, 133-139 (1984)

15. Bugeaud, Y: Bounds for the solutions of superelliptic equations. Compos. Math. 107, 187-219 (1997)

16. Poulakis, D: Solutions entières de l'équation $Y^{m}=f(X)$. Sémin. Théor. Nr. Bordx.) 3, 187-199 (1991)

17. Schmidt, WM: Integer points on curves of genus 1. Compos. Math. 81, 33-59 (1992)

18. Sprindžuk, VG: The arithmetic structure of integer polynomials and class numbers. Tr. Mat. Inst. Steklova 143, 152-174 (1977). Analytic number theory, mathematical analysis and their applications (dedicated to I.M. Vinogradov on his 85th birthday)

19. Voutier, PM: An upper bound for the size of integral solutions to $Y^{m}=f(X)$. J. Number Theory 53, $247-271$ (1995)

20. Bugeaud, Y, Győry, K: Bounds for the solutions of unit equations. Acta Arith. 74, 67-80 (1996)

21. Bugeaud, Y, Mignotte, M, Siksek, S: Classical and modular approaches to exponential Diophantine equations. I. Fibonacci and Lucas perfect powers. Ann. Math. 163, 969-1018 (2006)

22. Landau, E: Verallgemeinerung eines Pólyaschen satzes auf algebraische zahlkörper. Nachr. Ges. Wiss. Gött., Math.-Phys. Kl. 1918, 478-488 (1918)

23. Matveev, EM: An explicit lower bound for a homogeneous rational linear form in logarithms of algebraic numbers. II. Izv. Ross. Akad. Nauk, Ser. Mat. 64, 125-180 (2000)

24. Pethö, A, de Weger, BMM: Products of prime powers in binary recurrence sequences. I. The hyperbolic case, with an application to the generalized Ramanujan-Nagell equation. Math. Comput. 47, 713-727 (1986) 
25. Voutier, PM: An effective lower bound for the height of algebraic numbers. Acta Arith. 74, 81-95 (1996)

26. Bruin, N, Stoll, M: Deciding existence of rational points on curves: an experiment. Exp. Math. 17, 181-189 (2008)

27. Bruin, N, Stoll, M: The Mordell-Weil sieve: proving non-existence of rational points on curves. LMS J. Comput. Math. $13,272-306(2010)$

28. Flynn, EV: The Hasse principle and the Brauer-Manin obstruction for curves. Manuscr. Math. 115, 437-466 (2004)

29. Scharaschkin, V: Local-global problems and the Brauer-Manin obstruction. PhD thesis, University of Michigan (1999)

30. Bruin, N, Stoll, M: Two-cover descent on hyperelliptic curves. Math. Comput. 78, 2347-2370 (2009)

doi:10.1186/1687-1847-2013-245

Cite this article as: Pintér and Tengely: The Korteweg-de Vries equation and a Diophantine problem related to Bernoulli polynomials. Advances in Difference Equations 2013 2013:245.

\section{Submit your manuscript to a SpringerOpen ${ }^{\circ}$ journal and benefit from:}

- Convenient online submission

- Rigorous peer review

- Immediate publication on acceptance

- Open access: articles freely available online

- High visibility within the field

- Retaining the copyright to your article 